\title{
Donor Gluconate Rescues Livers from Uncontrolled Donation after Cardiac Death
}

\section{Ashley Limkemann ${ }^{1}$, Susanne L. Lindell ${ }^{1}$ Heather Reichstetter ${ }^{1}$, Valerie Plant $^{1}$, Dan Parrish ${ }^{1}$, Clementina Ramos ${ }^{1}$, Chris Kowalski ${ }^{1}$, Cristiano Quintini $^{2}$, and Martin J. Mangino ${ }^{1,3,4}$}

Departments of Surgery ${ }^{1}$, Emergency Medicine ${ }^{3}$, and Physiology and Biophysics ${ }^{4}$, Virginia Commonwealth University, Medical College of Virginia Campus, Richmond, VA 23298

And Department of Surgery ${ }^{2}$, Cleveland Clinic Foundation, Cleveland, OH 44195

\author{
Corresponding Author Contact \\ Martin J. Mangino, Ph.D. \\ Virginia Commonwealth University \\ Medical College of Virginia Campus \\ 1200 E. Broad St. \\ Richmond, VA 23298 \\ 804-628-3226 \\ 804-828-8300 (Fax) \\ mjmangino@vcu.edu
}

Funding: This work was supported by a grant from the National Institutes of Health R01 DK087737 and the Department of Defense W81XWH-12-1-0599 to Dr. Mangino. 


\begin{abstract}
Background: Ischemia from organ preservation or donation causes cells and tissues to swell due to loss of energy-dependent mechanisms of control of cell volume. These volume changes cause substantial preservation injury, because preventing these changes by adding cell impermeants to preservation solutions decreases preservation injury. The objective of this study was to assess if this effect could be realized early in uncontrolled donation after cardiac death (DCD) livers by systemically loading donors with gluconate immediately after death to prevent accelerated swelling injury during the warm ischemia period prior to liver retrieval.
\end{abstract}

Methods: Uncontrolled DCD rat livers were cold-stored in UW solution for 24 hours and reperfused on an isolated perfused liver (IPL) device for two hours or transplanted into a rat as an allograft for 7 days. Donors were pre-treated with a solution of the impermeant gluconate or a saline control immediately after cardiac death. Livers were retrieved after 30 minutes.

Results: In-vivo, gluconate-infused in donors immediately before or after cardiac death prevented DCD induced increases in total tissue water (TTW), decreased vascular resistance, increased oxygen consumption and ATP synthesis, increased bile production, decreased LDH release, and decreased histology injury scores after reperfusion on the IPL relative to saline-treated DCD controls. In the transplant model, donor gluconate pretreatment significantly decreased both ALT the first day after transplantation and total bilirubin the seventh day after transplantation.

Conclusions: Cell and tissue swelling plays a key role in preservation injury of uncontrolled DCD livers, which can be mitigated by early administration of gluconate solutions to the donor immediately after death. 


\section{INTRODUCTION}

Liver transplantation continues to be the treatment of choice for patients with end stage liver disease with the limiting factor being the availability of suitable liver grafts. One way to expand the donor pool is the use of livers donated after cardiac death (DCD). In DCD donors, death is declared on the basis of standard criteria of circulatory arrest (1). DCD can be divided into two categories: controlled and uncontrolled. Uncontrolled DCD donation occurs when patients sustain cardiopulmonary arrest prior to arrival at the hospital or failed resuscitation while in the hospital; controlled DCD donation occurs after planned withdrawal of care (2). Both categories of DCD, especially uncontrolled, are characterized by substantial warm ischemia injury not normally encountered with typical donation after brain death (DBD).

The most productive way to increase graft availability is to expand the donor pool to include donors that otherwise would not have been considered previously for liver donation. The greatest untapped pool of livers is uncontrolled DCD. Despite the potential for DCD to increase the number of livers available for transplantation, concerns exist regarding outcomes and the use of DCD liver allografts. DCD liver grafts have increased rates of primary non-function and early graft dysfunction compared to liver allografts from standard donation after brain death (DBD) (3). The amount of cold exposure during hypothermic liver preservation is related directly to the amount of preservation injury experienced by the graft after transplantation (4). Cold ischemia time is one of the single most important risk factor for liver preservation injury (5), which is potentiated with preexisting exposure to warm ischemia in DCD settings $(6 ; 7)$. Each hour of cold storage time is associated with a $6 \%$ increase in graft failure in DCD livers (8). Therefore, although brief periods of warm or cold ischemia may be safe for most livers, combinations of the two may become lethal at reperfusion $(9 ; 10)$, which limits the utility of DCD liver donation. As the quality of the donor liver decreases, a point will be reached where improvements in organ preservation will be required to maintain adequate graft function in the DCD group. Current preservation strategies are inadequate for uncontrolled DCD livers.

Ischemic injury to cells and tissues is generally based on the loss of cellular energetics. An important energy-based mechanism is cell swelling. As cells lose ATP due to ischemia, the sodium pump is disrupted, which allows sodium ions to enter the cell as they follow the electrochemical gradient. Chloride follows electrogenically and water then enters the cell osmotically. This cell swelling (not edema) produces membrane and mitochondrial injury and is a major cause of traditional hypothermic preservation injury in organ transplantation (11-13). Cell impermeants include sugars like sorbitol, 
gluconate, raffinose, lactobionate, and trehalose that prevent cell swelling. Anions like sulfate, phosphate, and cations like histidine (at physiological $\mathrm{pH}$ ) are also impermeants, because they are small enough to escape the microcirculation but too large or too charged to enter the cell. Thus, they preferentially load into the interstitial space, where they create a selective osmotic gradient opposing movement of water into the cell. Modern organ preservation solutions, like UW solution, HTK, and others, are effective largely because they contain cell impermeants (raffinose, lactobionic acid, gluconate, sulfate, phosphate for UW solution, and histidine and mannitol for HTK). Cell swelling occurs during warm ischemia too and at a much faster rate (14).

Organ preservation solutions were designed for use in single or small groups of organs and not for systemic administration per se. To achieve an effective interstitial concentration of an organ preservation component (like an impermeant) in donors using organ flush solution, it would require a large blood volume replacement, which is not practical. In contrast, a concentrated solution containing an effective non-toxic component (like an impermeant) can be given I.V. as a drug rather than as an arterial flush. This non-invasive approach could achieve extracellular therapeutic impermeant levels rapidly and equivalent to those found in organs after invasive, in-situ flushing with conventional organ preservation solutions. Gluconate is a cell-impermeant that is used currently in University of Wisconsin Machine Perfusion Solution, which is readily available, non-toxic, and easy to concentrate in solution, thereby minimizing the volume required to give the donor systemically to achieve an effective interstitial concentration. Therefore, the objective of the study was to examine the role of organ swelling in uncontrolled DCD liver preservation injury and to describe a novel cell impermeant-based therapeutic approach to the problem. The concept was to start the impermeant effect (seen with UW like solutions) with a concentrated gluconate solution administered systemically early in the uncontrolled DCD process to donors to prevent cell and tissue swelling injury before liver retrieval.

\section{MATERIALS AND METHODS}

All animal work was conducted under a protocol approved by the Virginia Commonwealth University Institutional Animal Care and Use Committee, which is governed by the rules and regulations set forth in the National Institute Health guide and the United States Department of Agriculture. 
Rat Liver DCD Model for Isolated Perfused Liver (IPL): Adult male Lewis rats were anesthetized with Ketamine/Xylazine (100mg/10mg/kg I.P), and the liver was exposed by a transverse incision. Males were used for vascular access (penile vein) to facilitate blood draws and infusions in the transplant study, and therefore, were used across studies for consistency. The bile duct was cannulated with a PE 10 catheter and the portal vein isolated. Phentolamine $(0.2 \mathrm{mg} / \mathrm{kg})$, heparin $(1 \mathrm{U} / \mathrm{g})$, gluconate, or vehicle (PBS) was given immediately before cardiac death as a proof of concept experiment to ensure distribution of the impermeants within the microcirculatory fluid compartments and to eliminate mixing as a factor by using the heart to test the impermeant effect. The rats were then killed by suffocation after bilateral pneumothorax. Impermeant solutions (15\%) or vehicle were infused I.V. over 10 min in a volume equal to $20 \%$ of the calculated blood volume. The animals remained untouched for 30 min after cardiac death to simulate an uncontrolled DCD period. The portal vein was cannulated with an 18 -gauge Angiocath and the liver flushed in-situ with 30-ml of cold UW solution (Bridge to Life, Inc., Chicago, IL). The liver was stored in UW solution on ice for $24 \mathrm{~h}$, after which, preservation injury was assessed by reperfusion on the IPL for $60 \mathrm{~min}$ at $37^{\circ} \mathrm{C}$. Greater reperfusion times are not possible when using this model for IPL, because we have found that the preparation develops edema and is not stable (unpublished data). In a subsequent study, impermeants were given after cardiac death to simulate clinical, uncontrolled DCD rules. In this setting, CPR was administered during the impermeant infusion to circulate the drug. Otherwise, the two recoveries were the same. Figure 1 shows the protocol for the two IPL studies and the one transplant study described below.

Rat Liver DCD Model for Orthotopic Liver Transplantation: Adult male Brown Norway rats were anesthetized as above. A stent was placed in the bile duct and secured with a 6-0 silk suture. Either PBS (vehicle, control) or 15\% Gluconate (impermeant) were administrated over $10 \mathrm{~min}$ as described previously. Thirty min after the cessation of heartbeat (warm ischemia), the livers were flushed with cold UW-solution and placed on ice for cuff preparation of the infrahepatic vena cava and portal vein. The livers were cold-stored in UW for $20 \mathrm{~h}$. In a separate group of animals the livers were cold stored for $20 \mathrm{~h}$ without the $30 \mathrm{~min}$ of warm ischemia (no DCD). The livers were then transplanted orthotopically into syngeneic recipient rats using standard surgical techniques as described previously $(16 ; 17)$. Blood samples were obtained from the penile vein on post-operative days 1, 3, and 7 for analysis of liver function and hepatocellular injury. After seven days, the animals were killed. Each liver transplant group contained the following replicates: No DCD Ischemia, $n=5$ attempted, 5 completed, no technical losses; DCD saline group, $\mathrm{n}=10$ attempted, 8 completed, 2 technical losses; and DCD gluconate group, $\mathrm{n}=11$ attempted, 8 completed, 3 technical losses. 
Tissue Distribution of Impermeant with cardiopulmonary resuscitation (CPR): An experiment was performed to evaluate the effectiveness of open cardiac massage (CPR) to circulate impermeants for tissue distribution in non-heart beating donors. A $15 \%$ gluconate solution containing a FITC-labeled impermeant marker was infused for the standard 10 min during CPR as described previously. After infusion and CPR, samples of tissues from skeletal muscle, left ventricle, lung, liver, ileal mucosa, and renal cortex were removed and ground in a Dounce homogenizer and sonicated. The homogenate was centrifuged at 12,000 x g for 5 min and FITC activity was assessed on a fluorescence plate reader (Biotek, Winooski, VT) using excitation / emission wavelengths of 490 and $525 \mathrm{nM}$, respectively. Measurements were made first with infusions of impermeants before cardiac death to give a baseline maximal or optimal distribution with cardiac mixing and then compared to values obtained during CPR after cardiac death in a paired companion rat. This was repeated 3 times. Data were expressed as [fluorescent material in the tissue before CPR / fluorescent material in the tissue after CPR] x 100.

Experimental Design: In the DCD rat liver, cold-storage studies evaluated by IPL, 4 groups of rat livers were used with 6 animals per group: 1.) non-preserved livers (Sham, negative control), 2.) DCD livers (positive controls), 3.) DCD + gluconate-treated livers (given before cardiac death), and 4.) DCD + gluconate-treated livers (given after cardiac death with CPR), There were 3 groups of rats in the transplant study that included: 1.) cold storage group (UW solution, no prior warm ischemia), 2.) DCD + saline group (30 min of warm ischemia, then $20 \mathrm{~h}$ of cold storage in UW solution) receiving a saline solution, and 3.) DCD + gluconate group (30 min of warm ischemia, then $20 \underline{\mathrm{h}}$ of cold storage in UW solution) receiving a $15 \%$ gluconate solution immediately before cardiac death.

Histology: Tissue was fixed in 10\% neutral buffered formalin, embedded in paraffin, sectioned to $4 \mu$, deparaffined, and stained with H \& E. The histology scores of liver tissue sections were blindly determined by one of the co-authors (Clementina Ramos) according to the scoring criteria described by Li (18) with modifications. Four attributes of liver injury (cellular vacuolization, vascular congestion, necrosis, and cytoplasmic condensation) were each given a score of 0-3 according to the criteria described in the Table. The aggregate scores for all categories were used to define injury as no change, moderate, intermediate, and severe, according to the Table. The aggregate scores were used in the nonparametric statistical analysis. 
Statistical Analysis: Descriptive statistics were calculated from the raw data, and all data were checked for distribution normality. All data were expressed as the group mean \pm SD. Multiple group comparisons were made by performing parametric ANOVA followed by a multiple comparison post-test (Bonferonni). Histologic assessment scores were analyzed by nonparametric ANOVA (Kruskal-Wallis test) and a multiple comparison test (Dunn test). A p value of 0.05 or less was considered statistically significant.

\section{RESULTS}

Cell swelling associated with DCD preservation injury is shown in Figure 2. Preservation injury of the whole liver due to DCD significantly increased total tissue water in liver biopsies recovered after reperfusion by $45 \%$. Treatment of donor rats before liver recovery with gluconate prevented completely cell and tissue swelling in the DCD livers after reperfusion. These data verify the desired biophysical effect of the impermeants used in this study and demonstrate clearly that the impermeants also are effective when given after cardiac death with CPR mixing.

Vascular resistance in the portal circulation significantly increased three fold after reperfusion in DCD livers, which normalized completely when gluconate was given at the time of cardiac death (Figure 3a). Figure $3 \mathrm{~b}$ illustrates the changes in $\mathrm{VO}_{2}$ by the liver in response to reperfusion after DCD recovery and cold storage and the response to gluconate. Specifically, $\mathrm{VO}_{2}$ significantly fell in the DCD group, relative to the sham group (negative control) but was restored when gluconate solution was given to the donor.

Bile production by the livers during the 60 minute reperfusion period is shown in Figure $3 \mathrm{c}$. Bile production was significantly decreased in the DCD group to nearly zero. Six of eight DCD livers failed to produce any bile during that period. Treatment with gluconate solution in the DCD donor significantly increased bile production at reperfusion compared to the untreated DCD livers by as much as 12 fold. Furthermore, the density of the bile formed in DCD livers recovered from gluconate pretreated donors after reperfusion was the same as the bile formed in fresh control livers recovered from donors with no prior warm ischemia or cold preservation $(1.075 \mathrm{mg} / \mathrm{ml} \pm 0.04$ for DCD livers from gluconate treated donors vs $1.085 \mathrm{mg} / \mathrm{ml} \pm 0.04$ for fresh livers). 
Figure $3 \mathrm{~d}$ shows release of LDH into the perfusate significantly increasing 35 fold in response to DCD preservation injury in the untreated livers. The low levels of LDH release into the perfusate in the sham livers was not different than the release observed in the DCD livers treated with the gluconate solution. In all variables in this study (Figure 3a-d), gluconate given after death by CPR mixing performed as well as the proof of concept group where gluconate mixing was ensured by administration immediately prior to cardiac death.

Tissue ATP levels and energy charge are shown in Figure 4. Intracellular ATP concentrations significantly decreased (72\%) in DCD liver tissue after reperfusion compared to sham liver controls. Gluconate significantly increased ATP levels after reperfusion, relative to the other DCD groups but were still significantly less than the ATP concentrations in the sham livers (Figure 4a). Similar changes and patterns were seen when the data were expressed as energy charge (Figure $4 \mathrm{~b}$ ).

Histologic signs of tissue injury in the livers after 60 minutes of IPL reperfusion were assessed by light microscopy of H \& E stained, paraffin-embedded liver sections (Figure 5). Histologic injury scores were significantly greater in the untreated DCD livers, relative to the fresh recovered livers or the DCD livers treated with gluconate. Representative sections from each group are shown and are characterized by the amount of necrosis, vascular congestion, cellular vacuolization, and cytoplasmic condensation present. The graph in Figure 5 summarizes the overall histological scores in each group, as defined by criteria summarized in the Table.

Plasma ALT and total bilirubin levels were measured for a week after liver transplantation in recipients that received a liver graft with 20 hours of cold storage (no DCD), DCD livers treated with saline (control), and DCD livers treated with gluconate (Figure 6). The effects of warm ischemia per se on preservation injury can be seen with the ALT levels $24 \mathrm{~h}$ after transplantation, which were six fold greater than the control group without prior warm ischemic exposure (Figure 6a). Administration of gluconate to DCD donors significantly decreased the ALT values at day one after transplantation by more than half. A week after transplantation, the total bilirubin concentration in the plasma increased $300 \%$ in the DCD group, relative to the control group without DCD. This DCD-induced increase in plasma bilirubin at one week was prevented completely by administration of gluconate to the donors immediately after death (Figure 6b). Recipient one-week survival was not significantly different in the gluconate-treated group (71\% vs 57\%). 
Finally, gluconate was given to DCD rats after cardiac death using CPR to circulate the impermeants. The tissue distribution of a fluorescently labeled impermeant given during CPR after cardiac death showed tissue impermeant levels that were generally the same and often greater than concentrations observed when the impermeant was delivered without CPR before cardiac arrest (Figure 7).

\section{DISCUSSION}

The shortage of usable donor livers is the limiting factor in treating patients in end stage liver disease with liver allotransplantation. Therefore, we devised a clinically useful and feasible method to intervene during the warm ischemic period in liver uncontrolled DCD after the declaration of death to decrease preservation injury. The role of cell and tissue swelling in preservation injury of uncontrolled DCD livers was explored by using loading of the cell impermeant gluconate in the donor prior to organ retrieval.

Gluconate solutions reversed DCD induced cell swelling and significantly attenuated preservation injury, which suggests that cell swelling plays a critical role in DCD liver preservation injury, as it does in conventional cold storage-induced preservation injury (11). Because the methods for assessing total tissue water (TTW) and thus cellular swelling cannot technically differentiate between cell swelling per se (intracellular water) versus tissue edema (interstitial water) or a combination of the two, we must use cell swelling in a broader context to include all tissue water including edema. The ischemia-induced changes in TTW were all abolished with gluconate pretreatment and the distribution of gluconate includes all extracellular spaces. Therefore, it is reasonable to suggest that the normalizations in TTW in gluconate-treated DCD livers were attributable mainly to changes in intracellular water (cell swelling) and not interstitial water (edema), because gluconate is a cell impermeant freely capable of escaping the capillary space, thereby creating an extracellular osmotic gradient but not an intravascular gradient.

Cell swelling affects the individual cell and the whole organ or tissue. As the cell swells during ischemia, the swelling causes injury of the plasma and mitochondrial membranes and rupture (22;23), especially after having been weakened by concomitant disassembly of key cytoskeletal proteins during ischemia $(20 ; 21 ; 24)$. As the parenchymal cells in the liver (mainly hepatocytes) swell, they compress the sinusoidal vascular spaces in aggregate, which decreases the diameter of the sinusoids and increases resistance to blood flow. Likewise, cell swelling of the sinusoidal endothelial cells lining the hepatic 
sinuses during warm ischemia further decreases the radius of the sinusoids and also increases resistance to flow $(25 ; 26)$. These features are partly responsible for the "no reflow" phenomenon, where blood flow resumes slowly or not at all after reperfusion. Therefore, one major secondary consequence of decreasing cell swelling in DCD livers is to prevent or attenuate no reflow at reperfusion. In fact, vascular resistance at reperfusion in this study tripled after DCD-induced preservation injury in untreated DCD donors but normalized completely in gluconate-treated donors. This is supportive evidence that prevention of cell swelling with prior gluconate administration in DCD donors prevents or reverses large increases in vascular resistance at reperfusion, probably by decompressing the sinusoids through the normalization of cell volume of both the parenchymal and sinusoidal endothelial cells. The increased oxygen delivery observed in gluconate-treated DCD donor livers at reperfusion is also consistent with these mechanisms, because this could increase the number of open sinusoids or flow through them, which would favor oxygen transfer out of the microcirculation. In fact, IV impermeants increase capillary blood flow, decrease cell swelling of tissues, and decrease lactate accumulation in rodents bled to $35 \mathrm{~mm} \mathrm{Hg}$ until a 55\% blood loss was reached (27). Another possible benefit from better microcirculation with impermeant loading is a more effective flush out at organ retrieval. Better perfusion increases delivery and equilibration of the UW preservative to flush homogenously and cool the liver. In the control DCD donor animals, swollen areas of the liver with compressed sinusoids do not perfuse well during retrieval, are not protected by the UW solution because of lack of entry into the tissue, and therefore, suffer local cold ischemia that causes greater reperfusion injury of the whole liver at transplantation.

The amount of bile production immediately after reperfusion or transplantation is one of the most important outcomes of preservation injury in liver grafts (28) and is used clinically as a good sign of early graft function. This is because bile production is a complex process that requires both anatomic and metabolic integrity. Anatomic structural integrity is needed to move bile out of the hepatocytes and into the biliary canaliculi and bile ducts. Because bile formation is also an active process, the metabolic system of the hepatocyte and cholangiocytes must be active, and those cells must be alive and anchored to their basement membranes. Processes, such as glycolysis, mitochondria respiration, and electron transport, are needed to make ATP at reperfusion, which drives the energetic work of bile formation and secretion. In the IPL studies, these processes seemed to be well preserved in DCD livers treated with gluconate, because bile formation increased from almost zero in the untreated DCD control livers to about $50 \%$ of normal in the treated groups. Furthermore, the specific gravity of the bile formed in the 
gluconate group was the same as the fresh controls, indicating that the bile was not simply an ultrafiltrate but that it may have had normal composition or levels of secreted products, like bile salts. Biliary function in DCD liver transplants with gluconate treatment was better than the saline controls because serum bilirubin values in the controls increased steeply after one week, which did not occur with gluconate treatment. This may depend on maintenance of structural integrity of the biliary system during ischemia or better microcirculatory perfusion of biliary structures (29).

In the liver transplant model, the hepatic artery was not revascularized. While this causes lethal injury to the liver in humans, it is tolerated for months in rats because the rat portal system communicates with the biliary arterial system to provide some oxygen delivery to the bile ducts and cholangiocytes $(30 ; 31)$. Nevertheless, a state of suboptimal oxygenation occurs resulting in terminal injury to the biliary system over time. In longitudinal studies, rats with liver transplants without arterialization develop bilomas and biliary sepsis and die 2-3 months after transplantation (unpublished observations). When the donor livers have prior warm ischemia, the process is accelerated greatly, and plasma bilirubin concentrations increase rapidly above normal in only a week after transplantation. This increase in serum bilirubin is prevented with prior gluconate loading, which may indicate that impermeants may be useful in preventing or lessening ischemic type biliary lesions after transplantation of DCD livers. This hypothesis however, has to be verified by well-designed histologic studies.

The cell impermeant solution used in the initial rat DCD model was administered immediately before cessation of the heartbeat in order to ensure mixing of the impermeants within the microcirculatory fluid compartments. This experimental design was used to eliminate mixing as a factor to test the impermeant effect per se and served as a proof of concept experiment. If this experiment failed to produce a positive effect, then there would be no reason to continue pre-clinical studies, because these conditions represent optimal conditions for solute mixing and distribution.

After studies confirm that impermeants improved DCD liver preservation injury under optimal conditions, mixing effects and details of donor administration become an important consideration. In current clinical protocols for liver DCD, the impermeant solutions can be administered only after death when the cardiovascular system is non-functional. Consequently, the impermeants need to be recirculated artificially to allow mixing and equilibration with the interstitial space. Closed or open chest CPR or extracorporeal membrane oxygenation (ECMO) are the most likely ways to accomplish this mixing and equilibration. In fact, we used 10 minutes of open chest CPR in this study to successfully 
mix and deliver the impermeants to the organs of interest, which supports the most likely clinical scenario. The tissue distribution and effectiveness of the impermeant in DCD liver preservation injury was equivalent to giving the impermeant immediately prior to cardiac death.

This experimental approach is clinically relevant, because it only entails the intravenous administration of about one liter of an impermeant solution to a DCD donor after declaration of death, which can be mixed effectively and delivered to organs using brief periods of CPR (10 minutes). This approach could be done easily in the emergency department, intensive care unit, or operating room prior to operative retrieval of the organ(s) to be transplanted to potentially salvage previously unusable grafts. Although not tested in this study, the impermeant solutions should be helpful to other recoverable organs also, because cell swelling and reversal by impermeants is a universal observation in organ preservation, and gluconate is regarded generally as non-toxic.

In conclusion, cell swelling during the warm ischemia phase of DCD donation of livers appears to play a major causal role in the preservation injury observed after reperfusion at transplantation. Our experimental work suggests that these events can be mitigated by intravenous administration of biophysically active cell impermeant to the donor at the time of cardiac death. Gluconate solutions produce strong results and should be considered for use in uncontrolled DCD liver donation in patients to expand the donor pool of usable livers for transplantations. Because early prevention of primary injury is key to successful organ preservation, early donor management is the next frontier in the use of extended criteria organs for preservation and utilization. 


\section{ACKNOWLEDGEMENTS}

This work was supported by a grant from the National Institutes of Health R01 DK087737 and the Department of Defense W81XWH-12-1-0599 to Dr. Mangino. 
Reference List

( 1) Bernat JL, D'Alessandro AM, Port FK, Bleck TP, Heard SO, Medina J, et al. Report of a National Conference on Donation after cardiac death. Am J Transplant 2006;6( 2) :281-91.

( 2) Foley DP, Fernandez LA, Leverson G, Chin LT, Krieger N, Cooper JT, et al. Donation after cardiac death: the University of Wisconsin experience with liver transplantation. Ann Surg 2005;242( 5) :724-31.

( 3) Merion RM, Pelletier SJ, Goodrich N, Englesbe MJ, Delmonico FL. Donation after cardiac death as a strategy to increase deceased donor liver availability. Ann Surg 2006;244(4) :555-62.

( 4) Furukawa H, Todo S, Imventarza O, Casavilla A, Wu YM, Scotti-Foglieni C, et al. Effect of cold ischemia time on the early outcome of human hepatic allografts preserved with UW solution. Transplantation 1991;51( 5 ) : 1000-4.

( 5) Stahl JE, Kreke JE, Malek FA, Schaefer AJ, Vacanti J. Consequences of cold-ischemia time on primary nonfunction and patient and graft survival in liver transplantation: a meta-analysis. PLoS One 2008;3( 6) :e2468.

( 6) Lee CY, Mangino MJ. Preservation methods for kidney and liver. Organogenesis 2009;5(3): 105-12.

( 7) Lindell S, Williams N, Brusilovsky I, Mangino MJ. Mouse IPK: A Powerful Tool to Partially Characterize Renal Reperfusion and Preservation Injury. Transplantation Journal 2011;5:15-22.

( 8) Mathur AK, Heimbach J, Steffick DE, Sonnenday CJ, Goodrich NP, Merion RM. Donation after cardiac death liver transplantation: predictors of outcome. Am J Transplant 2010;10( 11) :2512-9. 
( 9) Compagnon P, Lindell S, Ametani MS, Gilligan B, Wang HB, D'Alessandro AM, et al. Ischemic preconditioning and liver tolerance to warm or cold ischemia: experimental studies in large animals. Transplantation 2005;79( 10): :1393-400.

( 10) Lindell SL, Compagnon P, Mangino MJ, Southard JH. UW solution for hypothermic machine perfusion of warm ischemic kidneys. Transplantation 2005;79( 10) : 1358-61.

( 11) Belzer FO, Southard JH. Principles of solid-organ preservation by cold storage. Transplantation 1988;45( 4) : 673-6.

( 12) Southard JH, Belzer FO. Control of canine kidney cortex slice volume and ion distribution at hypothermia by impermeable anions. Cryobiology 1980;17(6) :540-8.

( 13) Southard JH, van Gulik TM, Ametani MS, Vreugdenhil PK, Lindell SL, Pienaar BL, et al. Important components of the UW solution. Transplantation 1990;49(2) :251-7.

( 14) Mees N, Southard JH, Belzer FO. Inhibition of ischemic induced cellular swelling in kidney cortex tissue by lactobionate anions. J Trauma 1982;22(2) :118-20.

( 15) Lindell SL, Southard JH, Vreugdenhil P, Belzer FO. Kupffer cells depress hepatocyte protein synthesis on cold storage of the rat liver. Transplantation 1994;58( 8) :869-74.

( 16) den Butter G, Lindell SL, Sumimoto R, Schilling MK, Southard JH, Belzer FO. Effect of glycine in dog and rat liver transplantation. Transplantation 1993:58:817-22.

( 17) Lindell SL, Hansen T, Rankin M, Danielewicz R, Belzer FO, Southard JH. Donor nutritional status--a determinant of liver preservation injury. Transplantation 1996;61( 2): :239-47.

( 18) Li W, Meng Z, Liu Y, Patel RP, Lang JD. The hepatoprotective effect of sodium nitrite on cold ischemia-reperfusion injury. J Transplant 2012;635179:1-10.

( 19) Compagnon P, Wang HB, Southard JH, Mangino MJ. Ischemic preconditioning in a rodent hepatocyte model of liver hypothermic preservation injury. Cryobiology 2002:44:269-78. 
( 20) Mangino MJ, Tian T, Ametani M, Lindell S, Southard JH. Cytoskeletal involvement in hypothermic renal preservation injury. Transplantation 2008;85(3) :427-36.

( 21) Tian T, Lindell SL, Henderson SC, Mangino MJ. Protective effects of ezrin on cold storage preservation injury in the pig kidney proximal tubular epithelial cell line ( LLCPK1) . Transplantation 2009;87( 10) :1488-96.

( 22) Buja LM, Entman ML. Modes of myocardial cell injury and cell death in ischemic heart disease. Circulation 1998;98( 14) :1355-7.

( 23) Steenbergen C, Hill ML, Jennings RB. Volume regulation and plasma membrane injury in aerobic, anaerobic, and ischemic myocardium in vitro. Effects of osmotic cell swelling on plasma membrane integrity. Circ Res 1985;57:864-75.

( 24) Tian T, Lindell SL, Lam M, Mangino MJ. Ezrin functionality and hypothermic preservation injury in LLC-PK1 cells. Cryobiology 2012;65(1): 1 :60-7.

( 25) Mazzoni MC, Borgstrom P, Intaglietta M, Arfors KE. Lumenal narrowing and endothelial cell swelling in skeletal muscle capillaries during hemorrhagic shock. Circ Shock 1989;29:27-39.

( 26) Mazzoni MC, Borgstrom P, Intaglietta M, Arfors KE. Capillary narrowing in hemorrhagic shock is rectified by hyperosmotic saline-dextran reinfusion. Circ Shock 1990;31:40718.

( 27) Parrish D, Lindell S, Reichstetter H, Aboutanos M, Mangino MJ. Cell impermeant based low volume resuscitation in hemorrhagic shock: A biological basis for injury involving cell swelling. Ann Surg. In press.

( 28) Furuyashiki S, Sumimoto K, Oku J, Kimura A, Fukuda Y, Dohi K, et al. The significance of bile secretion after the transplantation of long-preserved livers in the rat. Surg Today 1994;24( 1): $59-62$. 
( 29) Liu Q, Nassar A, Farias K, Buccini L, Baldwin W, Mangino M, et al. Sanguineous normothermic machine perfusion improves hemodynamics and biliary epithelial regeneration in DCD porcine livers. Liver Transpl 2014;20( 8) :987-999.

( 30) Lie TS, Hansen HH, Niehaus KJ. [Importance of graft arterialization in rat liver transplantation]. Langenbecks Arch Chir 1983;359( 2) :133-42.

( 31) Zhu XH, Pan JP, Wu YF, Ding YT. Effects of warm ischemia time on biliary injury in rat liver transplantation. World J Gastroenterol 2012;18( 43) :6308-14. 
Table 1. Histologic Scoring of Livers

\begin{tabular}{|l|l|l|l|l|}
\hline \multicolumn{5}{|l|}{ Damage or Change } \\
\hline & No Change & \multicolumn{1}{|c|}{ Moderate } & Intermediate & Severe \\
\hline Vacuolization & None & 1 or less/cell & $1 /$ Cell & $>1 /$ Cell \\
\hline Congestion & None & $<50$ RBCs/Field & $50-100$ RBCs & $>100$ RBCs \\
\hline Necrosis & None & 1 Cell/Field & $1-10$ Cells/Field & $>10$ Cells/Field \\
\hline $\begin{array}{l}\text { Cytoplasmic } \\
\text { Condensation }\end{array}$ & None & $<1 \%$ & $1-30 \%$ & $>30 \%$ \\
\hline VALUE & 0 & & & 3 \\
\hline
\end{tabular}

Final Score: 0, No Change; 1-4, Moderate; 5-8, Intermediate; >9, Severe 


\section{FIGURE LEGENDS:}

Figure 1: Diagram showing the experimental protocols for the three rat liver preservation experiments used in this study. For one group, donor impermeants (gluconate) were given immediately before cardiac death to allow for optimal mixing, followed by cold storage and IPL reperfusion to assess liver preservation injury. In the next experiment, donor impermeants were given immediately after cardiac death with CPR to allow for mixing and delivery of the impermeants to the organs of interest, followed by cold storage and IPL assessment of preservation injury. In the final group, impermeants were given before cardiac death and livers were assessed after cold storage by measuring liver outcomes using an orthotopic, syngeneic, rat liver transplant model with prolonged seven day survival after reperfusion. All three experimental groups share a surgical prep period, a DCD period (30 min warm ischemia), a cold storage period ( $24 \mathrm{~h}$ in cold UW solution), and an evaluation period ( $1 \mathrm{~h}$ on the IPL or 7-days after liver transplantation).

Figure 2: Total tissue water (TTW) accumulation in rat livers after DCD with $24 \mathrm{~h}$ of cold storage in University of Wisconsin solution, and $60 \mathrm{~min}$ of reperfusion on the isolated perfused liver (IPL) device. The livers were: Sham, DCD, DCD + gluconate (immediately prior to cardiac death), and DCD + gluconate-CPR (after cardiac death with CPR mixing). Values are mean \pm SD from 6 rats in each group. * $\mathrm{P}<0.05$ relative to all other values by ANOVA. Dashed line shows the average TTW value for the fresh group for reference.

Figure 3: Liver function assessed in rat livers after DCD with $24 \mathrm{~h}$ of cold storage in University of Wisconsin solution, and $60 \mathrm{~min}$ of reperfusion on the isolated perfused liver (IPL) device. The livers were: Sham, DCD, DCD + gluconate (immediately prior to cardiac death), and DCD + gluconate-CPR (after cardiac death with CPR mixing). Values are mean \pm SD from 6 rats in each group. $* \mathrm{P}<0.05$ relative to all other values by ANOVA. Panel A: Portal vascular resistance, Panel B: Oxygen uptake $\left(\mathrm{VO}_{2}\right)$, Panel C: Bile production, and Panel D: LDH release into the perfusate.

Figure 4: ATP (Panel A) and Energy Charge (Panel B) measured in rat liver tissue 60 min after reperfusion on the isolated perfused liver device (IPL) after DCD with $24 \mathrm{~h}$ of cold storage in University 
of Wisconsin solution. . The livers were: Sham, DCD, DCD + gluconate (immediately prior to cardiac death). Values are mean $\pm \mathrm{SD}$ from $4-8$ rats per group. $* \mathrm{P}<0.05$ between all groups, $\# \mathrm{P}<0.05$ relative to the other groups.

Figure 5: Histologic assessment by $\mathrm{H} \& \mathrm{E}$ stained, paraffin sectioned, rat livers using light microscopy for a sham liver (upper left), a liver after DCD (upper right), and a liver after DCD in a gluconate treated donor (lower right). Rat livers were obtained $60 \mathrm{~min}$ after reperfusion on the isolated perfused liver device (IPL) after DCD with 24 h of cold storage in University of Wisconsin solution. Graph inset: Summary of liver injury scores in the 3 groups, values are mean $\pm \mathrm{SD}$ from 6 rats per group. $* \mathrm{P}<0.05$, relative to the fresh and DCD + Gluconate groups.

Figure 6: Plasma ALT (panel A) and total bilirubin (panel B) values from rat liver transplant recipients after transplantation. The three groups include livers with $20 \mathrm{~h}$ of cold storage alone (no DCD), livers cold stored following 30 min of warm ischemia (DCD), and livers cold stored following 30 min of warm ischemia with gluconate given prior to warm ischemia (DCD 15\% gluconate). Values represent mean \pm $\mathrm{SD}, \mathrm{n}=6$ per group, $* \mathrm{P}<0.05$ between all groups (panel $\mathrm{A}$ ) and between DCD and the other groups (panel B).

Figure 7: The distribution of IV impermeants after cardiac death were determined in the DCD rat using open chest CPR to mix and deliver the solution to the organs and tissues. Dashed line is the $100 \%$ baseline for comparison. Values are mean of two independent pairs of experiments \pm SD. 
Figures

Fig 1

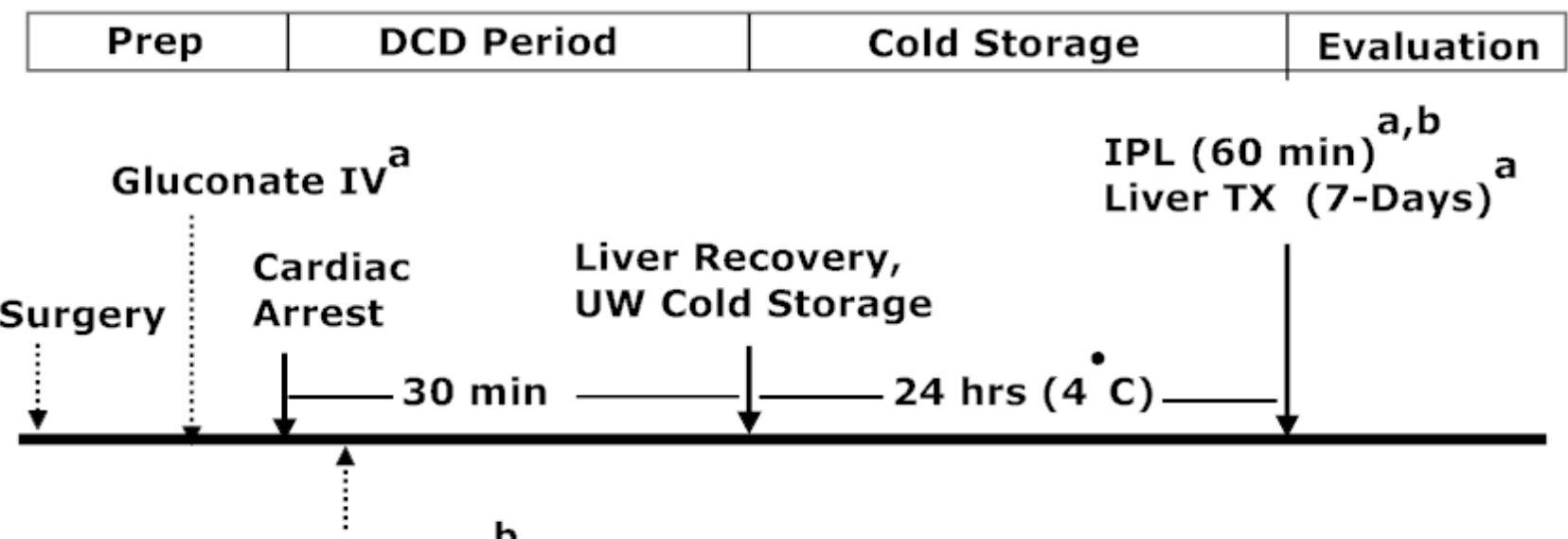

Gluconate IV ${ }^{\mathbf{b}}$

CPR $10 \mathrm{~min}$ 
Fig 2

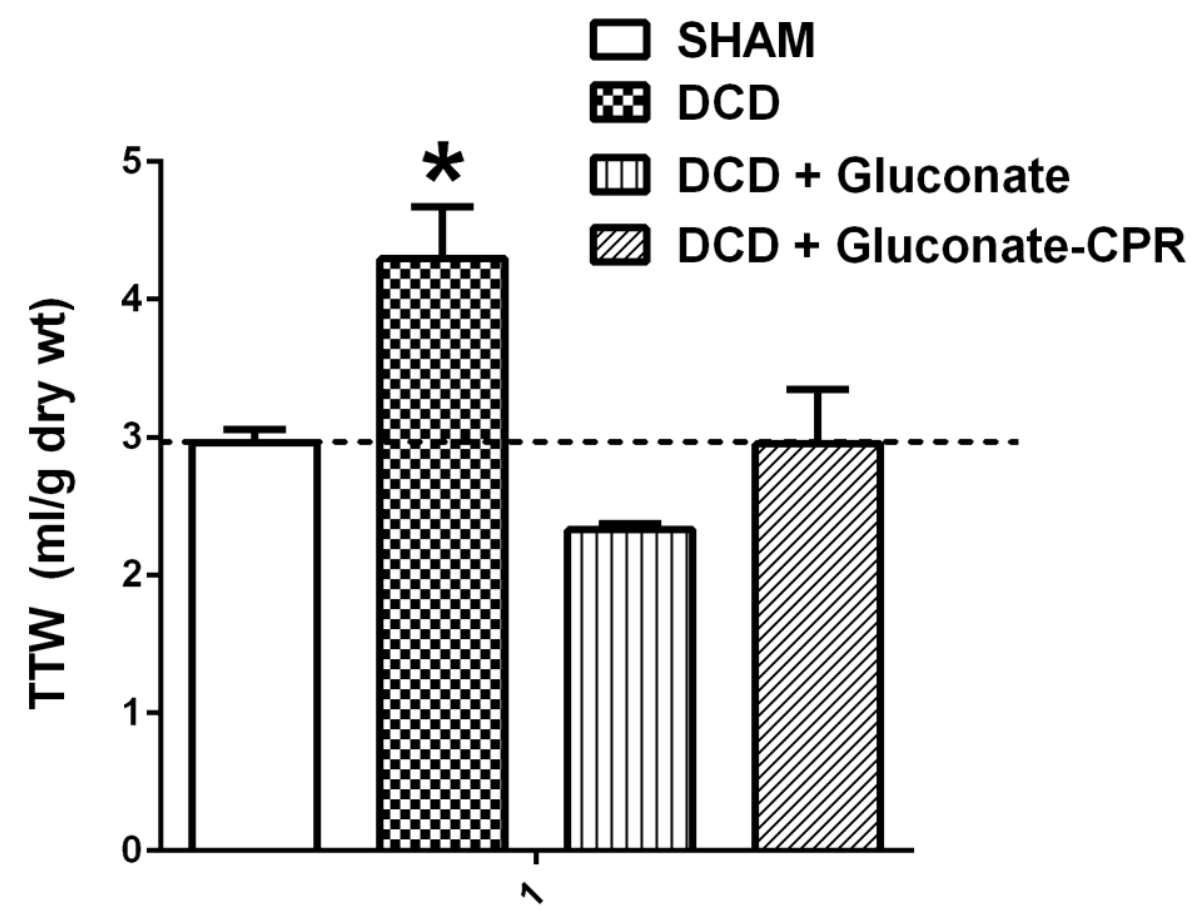


Fig 3

A.

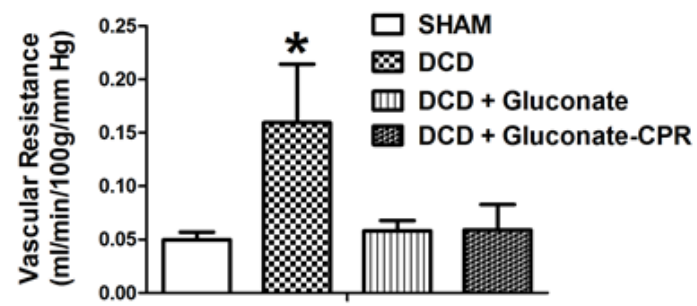

c.

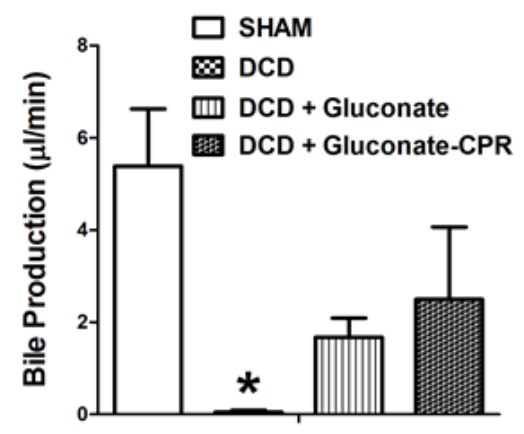

B.

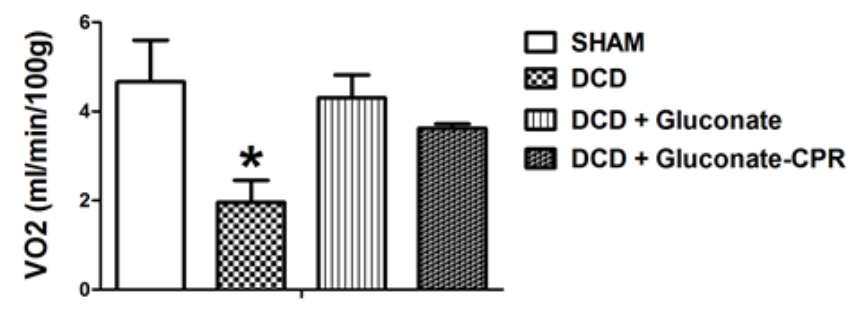

D.

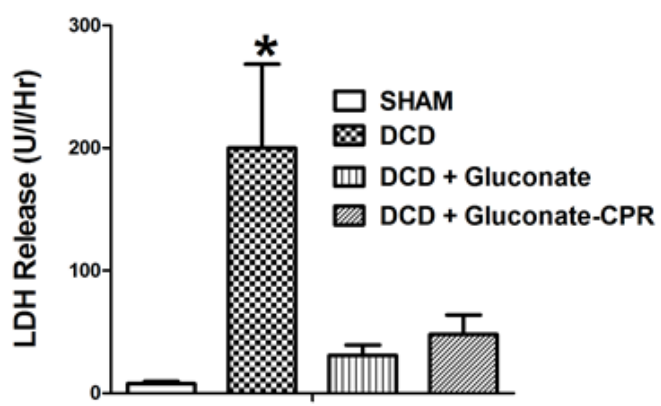


Fig 4

A.

B.

$\square$ Sham $\otimes D C D$ 四 DCD + Gluconate

$\square$ Sham $\otimes$ DCD 四 DCD + Gluconate
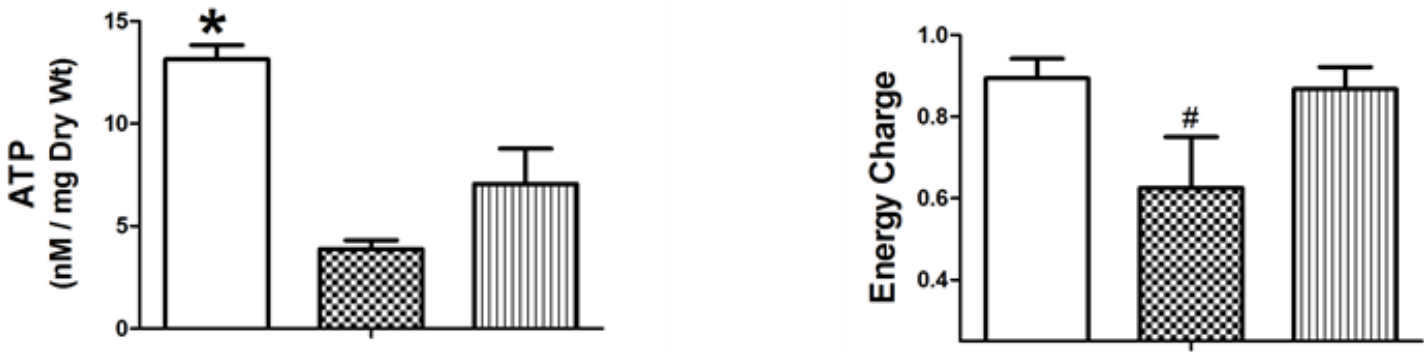
Fig 5

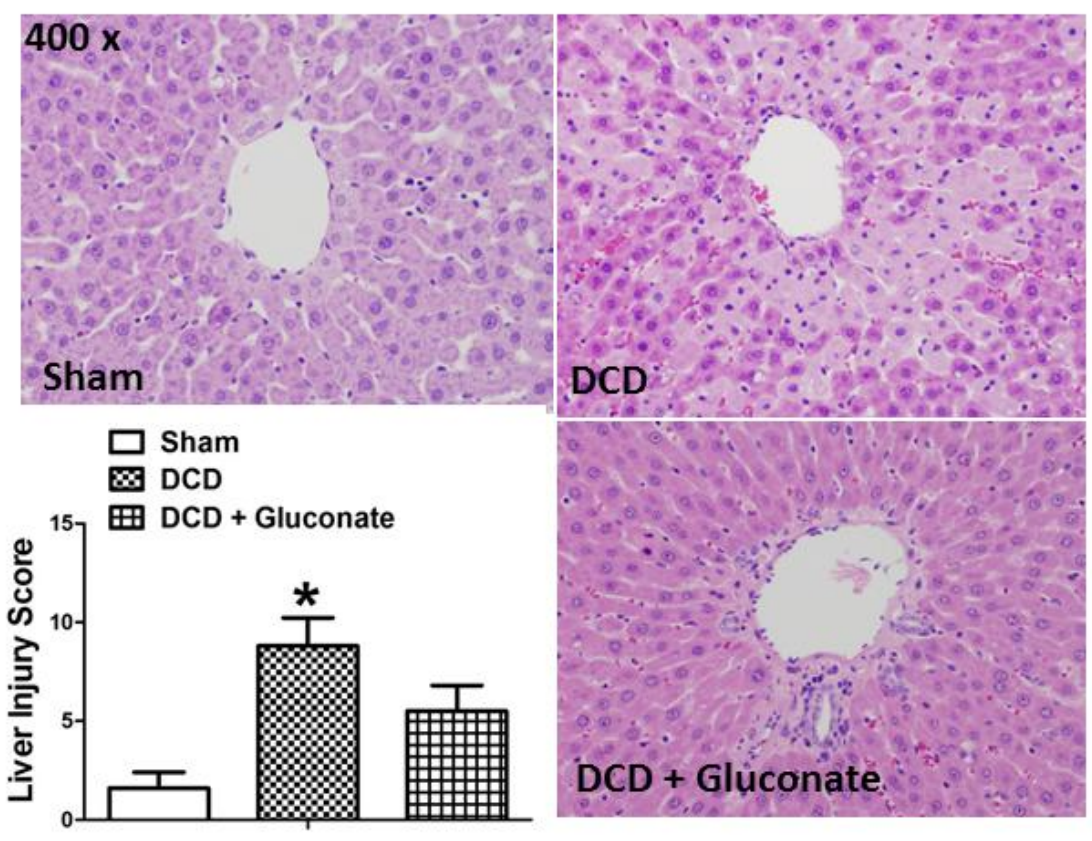


Fig 6

A

B
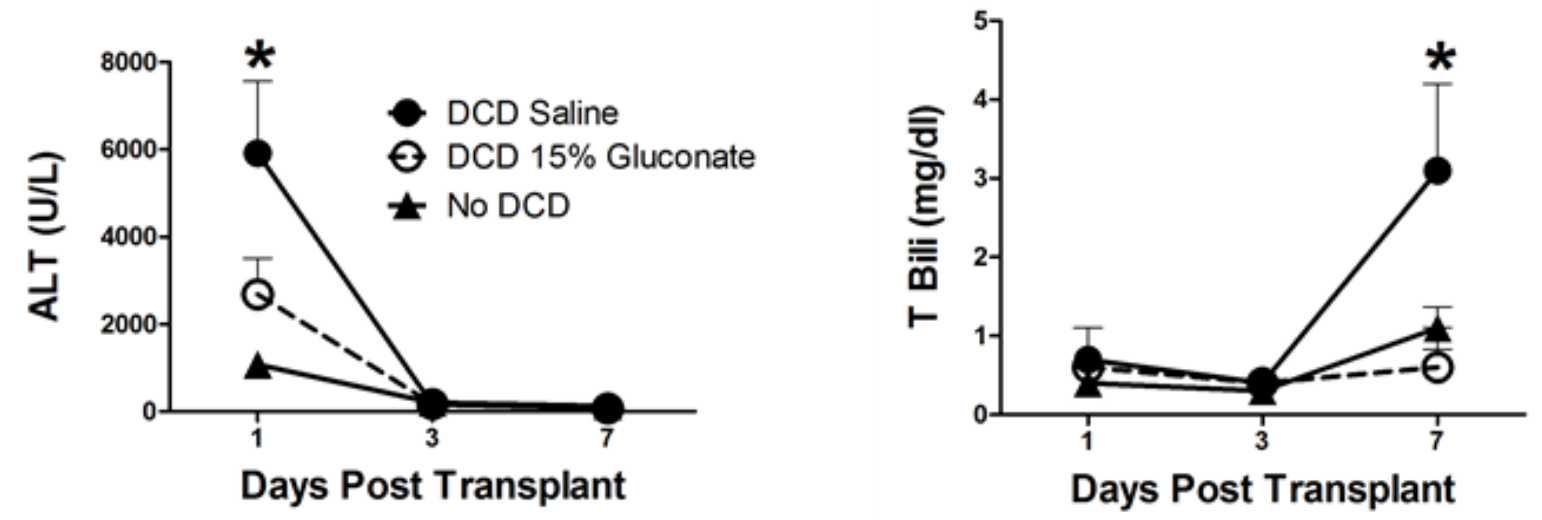
Fig 7

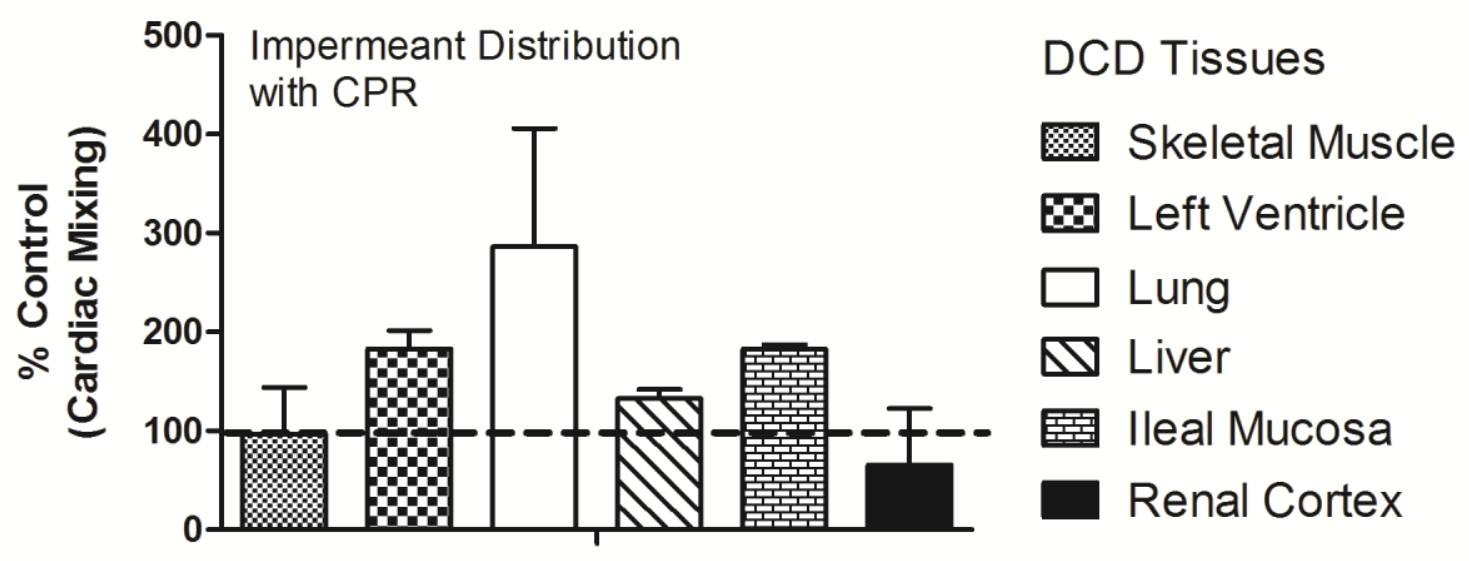

